\title{
LOCAL MAXIMA OF THE SAMPLE FUNCTIONS OF THE TWO-PARAMETER WIENER PROCESS
}

\author{
LANH TAT TRAN
}

ABSTRACT. It is shown that for almost all sample functions of the twoparameter Wiener process, the set of local maxima is dense in $R_{+}^{2}$.

Let $\left\{X(t): t \in R_{+}^{2}\right\}$ be the two-parameter Wiener process, that is a real valued Gaussian process with zero means and covariance

$$
\min \left(s_{1}, s_{2}\right) \min \left(t_{1}, t_{2}\right),
$$

where $s=\left(s_{1}, s_{2}\right), t=\left(t_{1}, t_{2}\right)$ are two points of $R_{+}^{2}$. Our purpose is to show that for almost all sample functions of $X$, the set of local maxima is dense in $R_{+}^{2}$. In the one-parameter case, almost all Brownian sample functions are monotone in no interval. As a consequence of this property, it can be shown that for almost all sample functions, the set of local maxima is dense in $R_{+}^{1}$. A proof is given in Freedman (1971). However, this method of approach does not provide a suitable proof in the two-parameter case due to the complex behavior of the sample functions.

The notation of Orey and Pruitt (1973) will be used. For $s=\left(s_{1}, s_{2}\right)$ and $t$ $=\left(t_{1}, t_{2}\right)$ with $s_{i} \leqslant t_{i}, i=1,2, X_{i=1}^{2}\left[s_{i}, t_{i}\right]$ is denoted by $\Delta(s, t)$ and $\Delta(t)$ in case $s=(0,0)$. Let $s, t \in R_{+}^{2}$. The variance of $X(t)-X(s)$ can be verified to be the two dimensional Lebesgue measure of $S(s, t)$ where $S(s, t)$ is the symmetric difference between $\Delta(s)$ and $\Delta(t)$. We will of ten state that $X$ has continuous sample functions, independent increments and denote the increments of $X$ over $\Delta(s, t)$ by $X(\Delta(s, t))$. For an account of these properties and further information on $X$, consult Kitagawa (1951), Chentsov (1956), Yeh (1960, 1963, a, b), Delporte (1966), C. Park (1969), W. J. Park (1970), Zimmerman (1972).

Definition. $X(\cdot, \omega)$ has a local maximum at $s$ if there is an open set $O$ containing $s$ such that $O \subset R_{+}^{2}$ and $X(t, \omega) \leqslant X(s, \omega)$ for all $t \in O$.

LEMMA 1. Let $s \in U$ where $U$ denotes the unit cube and $C \subset U$ be a cube with center at $s$, sides parallel to coordinate axes and equal to a. Let $I, \partial C$ be the interior and boundary of $C$. Then there exists a constant $\alpha>0$ and an $\varepsilon>0$ such that

Received by the editors October 20, 1975.

AMS (MOS) subject classifications (1970). Primary 60G15; Secondary 60G17.

Key words and phrases. Wiener process, local maxima, independent increments.

C American Mathematical Society 1976 


$$
P\left\{\sup _{t \in I} X(t)>\sup _{t \in \partial C} X(t)+a^{1 / 2}\right\}>\alpha,
$$

whenever $a<\varepsilon$.

Proof. Let $\left(u_{1}, u_{2}\right)$ and $\left(v_{1}, v_{2}\right)$ be the least and largest vertex of $C$. Then $s_{1}=\left(u_{1}+v_{1}\right) 2^{-1}, s_{2}=\left(u_{2}+v_{2}\right) 2^{-1}$. Define

$$
\begin{aligned}
& A_{1}=\left\{X\left(s_{1}, u_{2}\right)-X\left(u_{1}, u_{2}\right)>2 a^{1 / 2}\right\}, \\
& A_{2}=\left\{X\left(s_{1}, u_{2}\right)-X\left(v_{1}, u_{2}\right)>2 a^{1 / 2}\right\}, \\
& A_{3}=\left\{X\left(u_{1}, s_{2}\right)-X\left(u_{1}, u_{2}\right)>2 a^{1 / 2}\right\}, \\
& A_{4}=\left\{X\left(u_{1}, s_{2}\right)-X\left(u_{1}, v_{2}\right)>2 a^{1 / 2}\right\} .
\end{aligned}
$$

Now $A_{1}, A_{2}, A_{3}, A_{4}$ are independent because $X$ has independent increments. Pick $C$ with $a<\min \left(s_{1}, s_{2}\right)$; then the variances of

$$
\begin{array}{ll}
X\left(s_{1}, u_{2}\right)-X\left(u_{1}, u_{2}\right), & X\left(s_{1}, u_{2}\right)-X\left(v_{1}, u_{2}\right), \\
X\left(u_{1}, s_{2}\right)-X\left(u_{1}, u_{2}\right), & X\left(u_{1}, s_{2}\right)-X\left(u_{1}, v_{2}\right)
\end{array}
$$

are greater than $2^{-2} c a$, where $c$ is any positive number smaller than $s_{1}$ and $s_{2}$. Therefore,

$$
\begin{aligned}
P\left(A_{1} A_{2} A_{3} A_{4}\right) & =P\left(A_{1}\right) P\left(A_{2}\right) P\left(A_{3}\right) P\left(A_{4}\right) \\
& >\gamma \text { for some constant } \gamma .
\end{aligned}
$$

Let $A=A_{1} A_{2} A_{3} A_{4}$. Define

$$
M=\left\{\sup _{t \in I} X(t) \geqslant \sup _{t \in \partial C} X(t)+a^{1 / 2}\right\} .
$$

Let $\Delta(r, l) \subset U$ be any interval with both sides smaller than or equal to $a$. Clearly,

$$
\begin{aligned}
P(M) \geqslant & P\left(M / A \cap\left[\sup _{r, l \in U}|X(\Delta(r, l))|<a^{1 / 2}\right]\right) \\
& \cdot P\left(A \cap\left[\sup _{r, l \in U}|X(\Delta(r, l))|<a^{1 / 2}\right]\right) .
\end{aligned}
$$

For each $t \in \partial C$, construct a point $p^{t} \in I$ as follows: If $t$ is not a vertex of $C$, let $p^{t}$ be the point obtained from the perpendicular projection of $t$ onto the line passing through the center of $C$ and parallel to the side of $C$ on which $t$ lies. If $t$ is a vertex of $C$, pick $p^{t}$ to be the center $s$ of $C$. Now

$$
P\left\{\left[\inf _{t \in \partial C}\left(X\left(p^{t}\right)-X(t)\right)>a^{1 / 2}\right] / A \cap\left[\sup _{r, l \in U}|X(\Delta(r, l))|<a^{1 / 2}\right]\right\}=1 .
$$

It then follows that

$$
P\left(M / A \cap\left[\sup _{r, l \in U}|X(\Delta(r, l))|<a^{1 / 2}\right]\right)=1 .
$$

By (2), we obtain 


$$
P(M) \geqslant P\left(A \cap \sup _{r, l \in U}|X(\Delta(r, l))|<a^{1 / 2}\right) .
$$

But $P(A)>\gamma$ and $P\left(\sup _{r, l \in U}|X(\Delta(r, l))|<a^{1 / 2}\right)$ goes to 1 by Theorem 2.1 of Orey and Pruitt (1973). The proof of the lemma is completed.

LEMMA 2. Let $\left\{C_{n}\right\}$ be a sequence of cubes with common center s, sides parallel to coordinate axes and $C_{n} \supset C_{n+1}$ for all $n \geqslant 1$. Corresponding to each cube $C_{n}$, let $M_{n}$ be defined as in (1). Suppose that the sides of $C_{n}$ are small enough so that $P\left(M_{n}\right)>\alpha$ for all $n \geqslant 1$. Let the sides of $C_{n}$ be $a_{n}$ and assume $a_{n} \rightarrow 0$ as $n$ $\rightarrow \infty$. Define

$$
S_{n}=\left\{\sup _{t \in I_{n}} X(t)<\sup _{t \in \partial C_{n}} X(t)+a_{n}^{1 / 2}\right\},
$$

where $I_{n}$ and $\partial C_{n}$ are, respectively, the interior and boundary of $C_{n}$. Define

$$
E_{m}=\bigcap_{i=1}^{m} S_{i}
$$

If $\mu$ is a constant $<1$, then there exists a $k>m$ such that

$$
P\left(M_{k} E_{m}\right)>\mu P\left(M_{k}\right) P\left(E_{m}\right) .
$$

Proof. Pick $C_{k}$ with $k>m$ and let $t \in R_{+}^{2}$. Since $X$ has independent increments and continuous sample functions, it is possible to write $X(t)$ $-X(s)$ as the sum of two independent random variables, say $H_{k}(t)$ and $J_{k}(t)$, chosen in such a way that $J_{k}(t)$ and $M_{k}$ are independent; also $H_{k}(t)$ converges to zero almost surely as $k$ goes to infinity. To do this, consider the symmetric difference $S(s, t)$ between $\Delta(s)$ and $\Delta(t)$. This symmetric difference is the union of two parts: $S(s, t) \cap S\left(t^{k}, s^{k}\right)$ and $S(s, t) \cap S\left(t^{k}, s^{k}\right)^{\prime}$, where ' denotes the complement and $t^{k}, s^{k}$ are the least and largest vertex of $C_{k}$. Each part can be considered as the union of at most two nonoverlapping intervals. It is now clear that $H_{k}(t)$ can be chosen so that it depends only on the increments over intervals that form $S(s, t) \cap S\left(t^{k}, s^{k}\right)$; and $J_{k}(t)$ can be chosen so as to depend only on increments over intervals that form $S(s, t) \cap\left[S\left(t^{k}, s^{k}\right)\right]^{\prime}$. Since $X$ has independent increments, $J_{k}(t)$ is independent of $H_{k}(t)$ and $M_{k}$. Pick $\varepsilon>0$. Define

$$
B=\left[\sup _{t \in U}\left|H_{k}(t)\right|<\varepsilon\right]
$$

Clearly,

$$
\begin{aligned}
P( & \left.M_{k} E_{m}\right) \geqslant P\left(M_{k} E_{m} B\right) \\
& \geqslant P\left(M_{k} \bigcap_{i=1}^{m}\left\{\sup _{t \in I_{i}}\left(X_{t}-X_{s}\right)<\sup _{t \in \partial C_{i}}\left(X_{t}-X_{s}\right)+a_{i}^{1 / 2}\right\} B\right) \\
& \geqslant P\left(M_{k} \bigcap_{i=1}^{m}\left\{\sup _{t \in I_{i}}\left(J_{k}(t)+H_{k}(t)\right)<\sup _{t \in \partial C_{i}}\left(J_{k}(t)+H_{k}(t)\right)+a_{i}^{1 / 2}\right\} / B\right) P(B) \\
& \geqslant P\left(M_{k} \bigcap_{i=1}^{m}\left\{\sup _{t \in I_{i}} J_{k}(t)<\sup _{t \in \partial C_{i}} J_{k}(t)+a_{i}^{1 / 2}-2 \varepsilon\right\} / B\right) P(B) .
\end{aligned}
$$


But both $B$ and $M_{k}$ are independent of

$$
\bigcap_{i=1}^{m}\left\{\sup _{t \in I_{i}} J_{k}(t)<\sup _{t \in \partial C_{i}} J_{k}(t)+a_{i}^{1 / 2}\right\}
$$

Therefore,

$$
\begin{aligned}
& P\left(M_{k} E_{m}\right) \\
& \quad \geqslant P\left(M_{k} / B\right) P\left(\bigcap_{i=1}^{m}\left\{\sup _{t \in I_{i}} J_{k}(t)<\sup _{t \in \partial C_{i}} J_{k}(t)+a_{i}^{1 / 2}-2 \varepsilon\right\}\right) P(B) .
\end{aligned}
$$

But $P\left(M_{k}\right)>\alpha$ for all $k \geqslant 1$; and $P(B) \rightarrow 1$ as $k \rightarrow \infty$ since the sample functions of $X$ are continuous. The proof is completed by picking $k$ sufficiently large and $\varepsilon$ sufficiently small.

THEOREM. For almost all sample functions of $X$, the set of local maxima is dense in $R_{+}^{2}$.

Proof. Let $s \in R_{+}^{2}$ with $s_{1}>0, s_{2}>0$. By Lemmas 1 and 2, we can pick a sequence of cubes $\left\{C_{n}\right\}$ with center at $s$ and $U \supset C_{n} \supset C_{n+1}$ for all $n \geqslant 1$ such that

$$
P\left(M_{m+1} E_{m}\right)>\mu P\left(M_{m+1}\right) P\left(E_{m}\right) \text { for all } m \geqslant 1 \text {. }
$$

Now

$$
\begin{aligned}
P\left(E_{m+1}\right) & =P\left(E_{m} M_{m+1}^{\prime}\right)=P\left(E_{m}\right)-P\left(E_{m} M_{m+1}\right) \\
& <P\left(E_{m}\right)(1-\mu \alpha)<P\left(E_{1}\right)(1-\mu \alpha)^{m} .
\end{aligned}
$$

Therefore $P\left(\cap_{m=1}^{\infty} S_{m}\right)=0$. Since $s$ is arbitrary and $C_{1}$ can be chosen arbitrarily small, the theorem follows.

REMARK. Other properties related to the local maxima of Brownian sample functions as presented in Freedman (1971) also hold in the two-parameter case. The proof of these properties follows the same line of argument used in the one-parameter case and therefore is not included here.

\section{REFERENCES}

1. N. N. Čencov (1956), Wiener random fields depending on several parameters, Dokl. Akad. Nauk SSSR 106, 607-609. (Russian) MR 17, 1101.

2. J. Delporte (1966), Fonctions aléatoires de deux variables presque surement à échantillons continus sur un domaine rectangulaire borné, $\mathrm{Z}$. Wahrscheinlichkeitstheorie und Verw. Gebiete 6, 181-205. MR 35 \#3723.

3. D. Freedman (1971), Brownian motion and diffusion, Holden-Day, San Francisco, Calif. MR 45 \#6074.

4. T. Kitagawa (1951), Analysis of variance applied to function spaces, Mem. Fac. Sci. Kyushu Univ. Ser. A. 6, 41-53. MR 13, 855.

5. S. Orey and W. E. Pruitt (1973), Sample functions of the N-parameter Wiener process, Ann. Probability 1, no. 1, 138-163. MR 49 \# 11646.

6. C. Park (1969), A generalized Paley-Wiener-Zygmund integral and its applications, Proc. Amer. Math. Soc. 23, 388-400. MR 39 \# 7058.

7. W. J. Park (1970), A multi-parameter Gaussian process, Ann. Math. Statist. 41, 1582-1595. MR 42 \#6925. 
8. J. Yeh (1960), Wiener measure in a space of functions of two variables, Trans. Amer. Math. Soc. 95, 433-450. MR 23 \# A2735.

9. (1963a), Cameron-Martin translation theorems in the Wiener space of functions of two variables, Trans. Amer. Math. Soc. 107, 409-420. MR 32 \#6565.

10. (1963b), Orthogonal developments of functionals and related theorems in the Wiener space of functions of two variables, Pacific J. Math. 13, 1427-1436. MR 28 \#2433.

11. G. Zimmerman (1972), Some sample function properties of the two-parameter Gaussian process, Ann. Math. Statist. 43, 1235-1246. MR 47 \#948.

Department of Mathematics, Indiana University, Bloomington, Indiana 47401 